\title{
A Memory-Efficient and High-Speed Sine/Cosine Generator Based on Parallel CORDIC Rotations
}

\author{
Shen-Fu Hsiao, Member, IEEE, Yu-Hen Hu, Fellow, IEEE, and Tso-Bing Juang, Member, IEEE
}

\begin{abstract}
The sine/cosine function generator is based on parallelization of the original CORDIC algorithm by predicting all the rotation directions directly from the binary bits of the initial input angle. Unlike previous approaches that require complicated circuits or exponentially increased ROM, our proposed architecture has a relatively simple prediction scheme through an efficient angle recoding. The critical path delay is also reduced by utilizing the predicted rotation directions to design an efficient multioperand carry-save addition structure.
\end{abstract}

Index Terms-CORDIC, microrotation angle recoding, multioperand carry-save addition, parallel sign prediction, sine/cosine function generator.

\section{INTRODUCTION}

$\mathbf{O}$ NE OF THE key components in direct digital frequency synthesizer (DDFS) system [1] is the sine/cosine function generator that computes binary representation of $\sin \theta$ and $\cos \theta$ to a precision of $N$ fractional bits is. In this letter, we propose a novel realization of a sine/cosine generator based on the CORDIC algorithm [2]. CORDIC is an arithmetic algorithm developed to compute various elementary functions through a series of iterations of a unified microrotations operation. In particular, in a circular rotation mode, $N$ microoperations as illustrated below will be executed for $i=1,2, \cdots, N$

$$
\left\{\begin{aligned}
x_{i+1} & =x_{i}+\sigma_{i} 2^{-i} y_{i} \\
y_{i+1} & =y_{i}-\sigma_{i} 2^{-i} x_{i} \\
z_{i+1} & =z_{i}-\sigma_{i} \tan ^{-1}\left(2^{-i}\right), \quad \sigma_{i}=\operatorname{sign}\left(z_{i}\right) \in\{1,-1\} . \\
& =z_{i}-\sigma_{i} \alpha_{i}
\end{aligned}\right.
$$

After $N$ iterations, the accumulated rotation angle is

$$
\theta=z_{1}-z_{N+1}=\sum_{i=1}^{N} \sigma_{i} \tan ^{-1}\left(2^{-i}\right) \equiv \sum_{i=1}^{N} \sigma_{i} \alpha_{i} .
$$

Using the definition of $\alpha_{i}$, one has (note that $\cos \sigma_{i} \alpha_{i}=\cos \alpha_{i}$ )

$$
\begin{aligned}
{\left[\begin{array}{l}
x_{i+1} \\
y_{i+1}
\end{array}\right] } & =\left[\begin{array}{cc}
1 & \sigma_{i} 2^{-i} \\
-\sigma_{i} 2^{-i} & 1
\end{array}\right]\left[\begin{array}{l}
x_{i} \\
y_{i}
\end{array}\right] \\
& =\frac{1}{\cos \alpha_{i}}\left[\begin{array}{cc}
\cos \sigma_{i} \alpha_{i} & \sin \sigma_{i} \alpha_{i} \\
-\sin \sigma_{i} \alpha_{i} & \cos \sigma_{i} \alpha_{i}
\end{array}\right]\left[\begin{array}{l}
x_{i} \\
y_{i}
\end{array}\right] .
\end{aligned}
$$

\footnotetext{
Manuscript received October 21, 2002; revised April 8, 2003. The associate editor coordinating the review of this manuscript and approving it for publication was Dr. Richard C. Kavanagh.

S.-F. Hsiao and T.-B. Juang is with the Department of Computer Science and Engineering, National Sun Yat-sen University, 807 Kaohsiung, Taiwan, R.O.C. (e-mail: sfhsiao@cse.nsysu.edu.tw; tsobing@garfield.cse.nsysu.edu.tw).

Y.-H. Hu is with the Department of Electrical and Computer Engineering, University of Wisconsin-Madison, Madison, WI 53706-1691 USA (e-mail: hu@engr.wisc.edu).

Digital Object Identifier 10.1109/LSP.2003.821705
}

Then, it can be easily deduced that

$$
\left[\begin{array}{l}
x_{N+1} \\
y_{N+1}
\end{array}\right]=\frac{1}{K}\left[\begin{array}{cc}
\cos \theta & \sin \theta \\
-\sin \theta & \cos \theta
\end{array}\right]\left[\begin{array}{l}
x_{1} \\
y_{1}
\end{array}\right]
$$

where $K=\prod_{i=1}^{N} \cos \alpha_{i}=\prod_{i=1}^{N}\left(1+2^{-2 i}\right)^{-1 / 2}$ is a constant that can be precomputed in advance. Set $x_{1}=K, y_{1}=0$, $z_{1}=\theta$, then $x_{N+1}=\cos \theta, y_{N+1}=\sin \theta$ can be easily computed after $N$ iterations. In conventional CORDIC, the direction $\sigma_{i}=\operatorname{sign}\left(z_{i}\right)$ is determined sequentially since it depends on the sign of $z_{i}$ calculated at the previous iteration. This dependence relation makes it difficult to execute multiple microrotations in parallel. In this letter, we propose a new method to quickly select the rotation directions $\left\{\sigma_{i}\right\}$ in order to speed up the calculation.

\section{PRedictions of Rotation DiRections}

\section{A. Binary to Bipolar Recoding (BBR)}

The initial input angle $\theta=\left(-\theta_{0}\right)+\sum_{j=1}^{N} \theta_{j} 2^{-j}$ with $\theta_{j} \in\{0,1\}$ is assumed to be in the range $|\theta|<\pi / 4$ as in the application example of DDFS. It has been shown in [3] that $\tan ^{-1} 2^{-i}=2^{-i}$ to $N$-bit precision if $i \geq m=\left\lceil\left(N-\log _{2} 3\right) / 3\right\rceil$. Thus, the last $2 N / 3$ rotation directions (from $\sigma_{m}$ to $\sigma_{N}$ ) can be obtained in parallel after completing the first $N / 3$ iterations. As proposed in [3], we divide the angle into two parts (the higher part and the lower part)

$$
\theta=\theta_{H}+\theta_{L}=\underbrace{\left(-\theta_{0}\right)+\sum_{j=1}^{m-1} \theta_{j} 2^{-j}}_{\theta_{H}}+\underbrace{\sum_{j=m}^{N} \theta_{j} 2^{-j}}_{\theta_{L}} .
$$

The binary bits $\theta_{j} \in\{0,1\}$ in the higher part $\theta_{H}$ can be recoded into bipolar digits as follows:

$$
\begin{aligned}
\theta_{H}= & \left(-\theta_{0}\right)+\sum_{j=1}^{m-1} \theta_{j} 2^{-j} \\
= & \left(-\theta_{0}\right)+\sum_{j=1}^{m-1}\left[2^{-j-1}+\left(2 \theta_{j}-1\right) 2^{-j-1}\right] \\
= & \left(-\theta_{0}\right)+2^{-1}+\sum_{k=2}^{m} r_{k} 2^{-k}-2^{-m} \\
& \quad \text { where } r_{k}=\left(2 \theta_{k}-1\right) \in\{1,-1\} .
\end{aligned}
$$

Equation (3) is called BBR for $\theta_{j}, j=0,1, \ldots, m-1$.

\section{B. Microrotation Angle Recoding (MAR)}

Since $\tan ^{-1}\left(2^{-i}\right) \neq 2^{-i}$ for $i=1, \cdots, m-1$, we decompose each positional binary weighting $2^{-i}, i=1, \cdots, m-$ 1 into the combination of significant $\tan ^{-1}\left(2^{-j}\right)$ terms plus 
an error term $e_{i}$ collecting all the other insignificant values of $\tan ^{-1}\left(2^{-j}\right), j>m$. For simplicity, we take $N=24$ as an example where $m=\left\lceil\left(N-\log _{2} 3\right) / 3\right\rceil=8$. The microrotation angle recoding from $2^{-i}$ to $\tan ^{-1}\left(2^{-i}\right), i=1, \cdots, 7$ is

$$
\begin{aligned}
2^{-1}= & \tan ^{-1}\left(2^{-1}\right)+\tan ^{-1}\left(2^{-5}\right)+\tan ^{-1}\left(2^{-8}\right) \\
& +\underbrace{0.000000000100111100001110_{2}}_{e_{1}} \\
2^{-2}= & \tan ^{-1}\left(2^{-2}\right)+\tan ^{-1}\left(2^{-8}\right) \\
& +\underbrace{0.000000000100100100010100_{2}}_{e_{2}} \\
2^{-3}= & \tan ^{-1}\left(2^{-3}\right)+\underbrace{0.000000000010101001000101_{2}}_{e_{3}} \\
2^{-4}= & \tan ^{-1}\left(2^{-4}\right)+\underbrace{0.000000000000010101010010_{2}}_{e_{5}} \\
2^{-5}= & \tan ^{-1}\left(2^{-5}\right)+\underbrace{0.000000000000000010101010_{2}}_{e_{6}} \\
2^{-6}= & \tan ^{-1}\left(2^{-6}\right)+\underbrace{0.000000000000000000010101_{2}}_{e_{7}} \\
2^{-7}= & \tan ^{-1}\left(2^{-7}\right)+\underbrace{0.000000000000000000000010_{2}} .
\end{aligned}
$$

The BBR for $\theta_{H}$ with $N=24$ is

$$
\theta_{H}=\left(1-2 \theta_{0}\right) 2^{-1}+\sum_{k=2}^{8} r_{k} 2^{-k}-2^{-8}
$$

The first eight rotation directions are selected concurrently as

$$
\begin{aligned}
\sigma_{1} & =\left(1-2 \theta_{0}\right) \\
\sigma_{k} & =r_{k}=\left(2 \theta_{k-1}-1\right), \quad k=2, \cdots, 8 .
\end{aligned}
$$

Then, all the signed error terms $\sigma_{i} e_{i}, i=1, \ldots, 7$ and the last term $-2^{-8}$ in (5) are added to $\theta_{L}$, generating the corrected lower part $\hat{\theta}_{L}$ represented in twos complement format, i.e.,

$$
\begin{aligned}
\hat{\theta}_{L} & =\theta_{L}+\sum_{i=1}^{7} \sigma_{i} e_{i}-2^{-8} \\
& =\left(-\hat{\theta}_{7}\right) 2^{-7}+\sum_{k=8}^{24} \hat{\theta}_{k} 2^{-k}, \quad \hat{\theta}_{k} \in\{0,1\}, k=8, \ldots, 24 .
\end{aligned}
$$

It can be shown that $\left|\hat{\theta}_{L}\right|<2^{-7}$. Since $\tan ^{-1} 2^{-i}=2^{-i}$, $i \geq 8$ within precision of 24 fractional bits, the algorithm converges after the above selection of directions for the first several rotations. The directions for the remaining microrotations can be derived immediately from (7) using again the BBR

$$
\begin{aligned}
\hat{\theta}_{L} & =\left(-\hat{\theta}_{7}\right) 2^{-7}+\sum_{k=8}^{24} \hat{\theta}_{k} 2^{-k} \\
& =\left(-\hat{\theta}_{7}\right) 2^{-7}+\sum_{k=9}^{25}\left(2 \hat{\theta}_{k-1}-1\right) 2^{-k}+2^{-8}-2^{-25} \\
& =\left(1-2 \hat{\theta}_{7}\right) 2^{-8}+\sum_{k=9}^{25} \hat{r}_{k} 2^{-k}-2^{-25} \\
\hat{r}_{k} & =\left(2 \hat{\theta}_{k}-1\right) \in\{1,-1\}
\end{aligned}
$$

leading to the parallel prediction

$$
\begin{aligned}
& \hat{\sigma}_{8}=\left(1-2 \hat{\theta}_{7}\right) \\
& \hat{\sigma}_{k}=\hat{r}_{k}=\left(2 \hat{\theta}_{k}-1\right), \quad k=9, \cdots, 25
\end{aligned}
$$

for the last $2 N / 3$ microrotations.

\section{IMPLEMENTATION AND COMPARISON}

Fig. 1(a) is a 24-bit sine/cosine generator based on unfolded CORDIC architecture where each numbered block (a stage) denotes a microrotation performing the recurrence of $x$ and $y$ in (1). Note that the microrotation of angle $\tan ^{-1}\left(2^{-5}\right)$ is repeated once and the microrotation of angle $\tan ^{-1}\left(2^{-8}\right)$ is repeated three times. The scaling factor is still kept constant by taking into account these fixed repetitions

$$
K^{\prime}=\prod_{i=1}^{N}\left(1+2^{-2 i}\right)^{-\frac{1}{2}} \times\left(1+2^{-10}\right)^{-\frac{1}{2}} \times\left(1+2^{-16}\right)^{-\frac{3}{2}} .
$$

This constant can be precomputed and serves as one of the initial inputs $x_{1}=K^{\prime}, y_{1}=0, z_{1}=\theta$.

Since the first $m$ bits of the input angle are directly used as the directions in the first $m$ rotations, the execution of stages 1-12 (including repetition stages) in the left of Fig. 1(a) can be performed in parallel with the prediction adder that generates the directions for the remaining microrotations. We adopt carry-save addition (CSA) in each stage where a 4:2 compressor is used to produce the carry-save form (a sum term plus a carry term) for each output, as shown in Fig. 1(b). Assuming the delay of a $4: 2$ compressor to be $2 T_{\mathrm{FA}}$ where $T_{\mathrm{FA}}$ is the delay of a full adder, the delay for the stages in the left of Fig. 1(a) is $20 T_{\mathrm{FA}}$. Note that the first stage does not need CSA. The prediction adder is to calculate the sum of the nine operands in (7), using a CSA tree and a fast carry-propagate adder (CPA), leading to a delay of $4 T_{\mathrm{FA}}+T_{\mathrm{CPA}}$ where $T_{\mathrm{CPA}}$ denotes the delay of a CPA. In general, the prediction adder is not in the critical path as long as a fast CPA is used.

The derivation in Section II can be easily extended to different bit precision $N$ where the total number of repetitions $l(N)$ can be found to be $l(16)=2, l(24)=4, l(32)=7$. In some application (such as the DDFS) where the input angle is further limited in $0 \leq \theta<\pi / 4$, the first rotation direction is always $\sigma_{1}=1$, and thus the first several stages controlled by $\sigma_{1}$ can be merged and precomputed along with the constant factor $K^{\prime}$ as the initial input to the $\mathrm{X} / \mathrm{Y}$ datapath. In this situation, the numbers of repetitions in the sine/cosine generation are reduced to $l(16)=1, l(24)=2, l(32)=4$.

The second half of microrotation stages can be merged into a multioperand carry-save addition architecture by observing that

$$
\left\{\begin{array}{l}
x_{k+l}=x_{k}+y_{k} \sum_{i=k}^{k+l-1} r_{i} 2^{-i} \\
y_{k+l}=y_{k}-x_{k} \sum_{i=k}^{k+l-1} r_{i} 2^{-i}
\end{array}, \quad k>\frac{N}{2}, \quad l=1,2, \ldots .\right.
$$

Thus, the microrotation stages numbered from 13-25 in Fig. 1(a) can be merged into a CSA tree performing the parallel addition of 28 operands (14 numbers in carry-save forms) with critical path delay $7 T_{\mathrm{FA}}+T_{\mathrm{CPA}}$. Summing up the delay of all the stages, the total delay of our proposed sine/cosine 


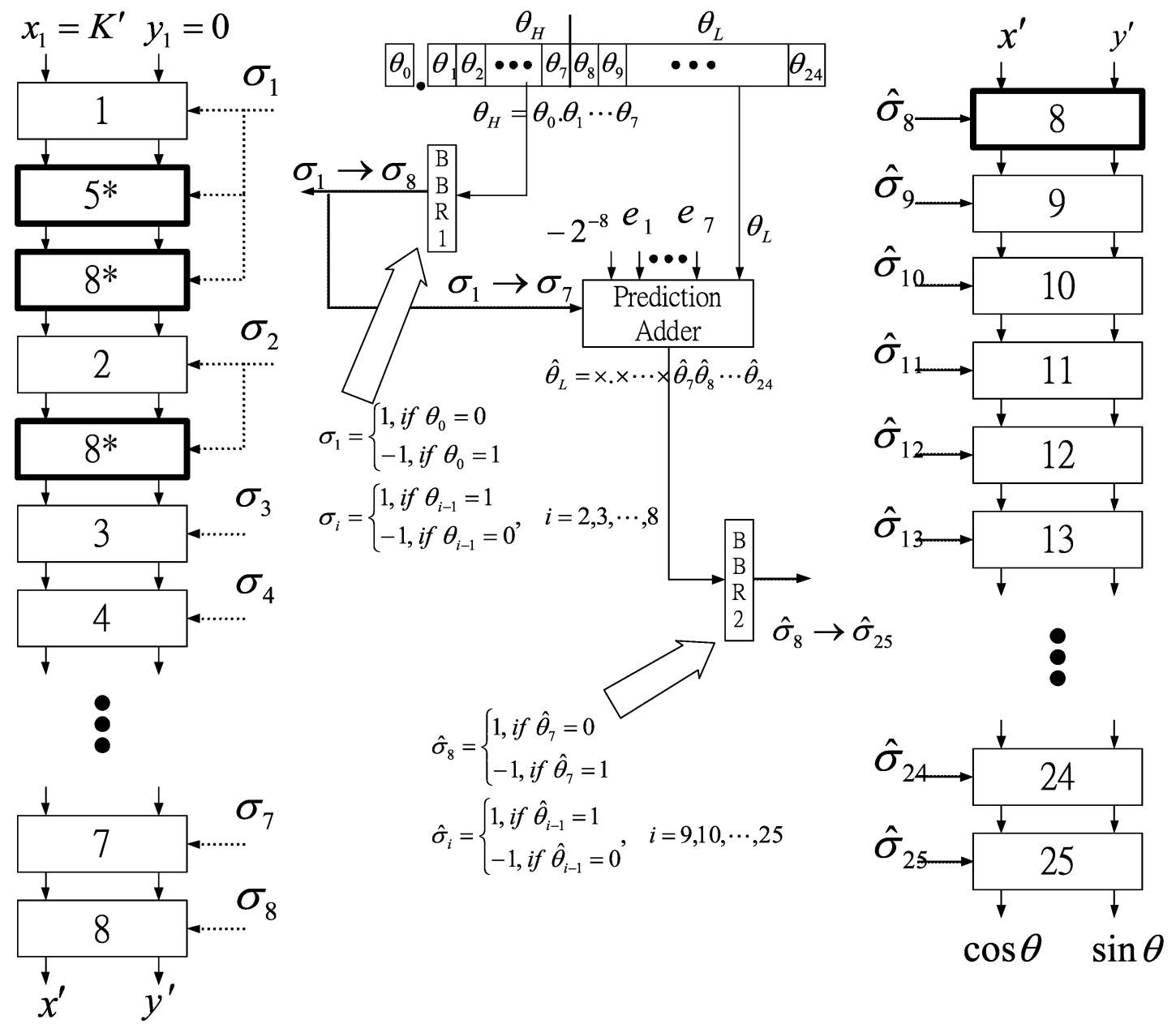

(a)

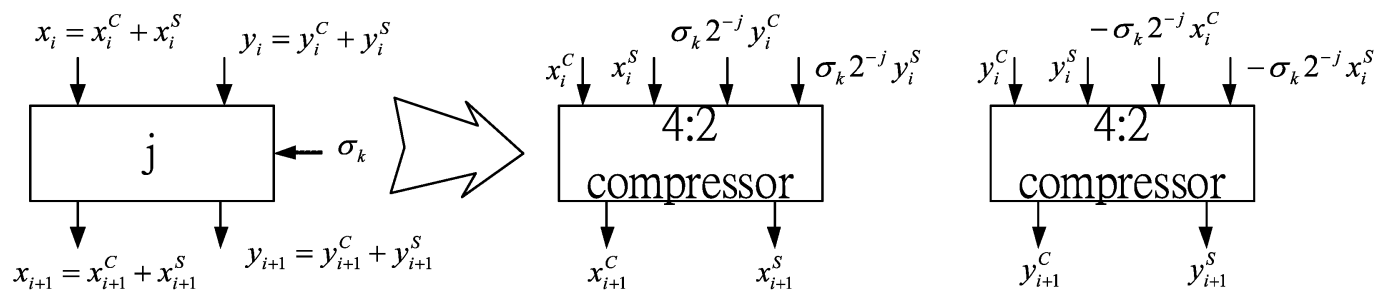

(b)

Fig. 1. (a) Architecture of a 24-bit sine/cosine generator based on parallel CORDIC rotations and (b) the carry-save addition implementation in a stage.

generator is $37 T_{\mathrm{FA}}+T_{\mathrm{CPA}}$ for 24-bit accuracy, a significant speed improvement compared with other previous approaches as will be discussed in the following.

Table I compares our proposed sine/cosine generator with other CORDIC rotation algorithms. To make a fair comparison, we assume that all methods use CSA in X/Y datapath except for the last stage where a fast CPA is required to obtain the nonredundant representation of the output ( $T_{\mathrm{CPA}}$ is not counted in Table I for reason of clarity). In [3], the rotation directions after $m$ iterations are derived from the $\mathrm{z}$ remainder. However, the first $m$ iterations still adopt the conventional sequential approach where a delay of $\left\lceil\log _{2} N\right\rceil \times T_{\mathrm{FA}}$ is assumed for an $N$-bit
CPA. In [4], two rotations are executed in a single step at the price of more complicated $Z$ datapath where several most significant digits are examined. In [5], the first $m$ rotation directions are predicted based on approximation of binary angle input to $\tan ^{-1} 2^{-i}$, similar to our method. But the direction prediction requires several carry-look-ahead adders plus complicated logic circuits instead of directly from the binary bits of the input angle as in our method. In [6], the first several directions are derived using a ROM of size exponentially increased with $N$. Unlike these above methods that require some delay to predict the first $m$ rotation directions, our proposed method generates the first $m$ rotation directions immediately from the first $m$ binary bits of 
TABLE I

COMPARISON OF $N$-Bit CORDIC-BASEd Sine/Cosine GENERATors With DifFERENT PREDICTION SCHEMES

\begin{tabular}{l|l|l|l|l|l|l|l}
\hline method & Delay (in units of $\left.T_{F A}\right)$ & $\begin{array}{l}\text { Delay } \\
(N=16)\end{array}$ & $\begin{array}{l}\text { Delay } \\
(N=24)\end{array}$ & $\begin{array}{l}\text { Delay } \\
(N=32)\end{array}$ & Z-path & ROM & $\begin{array}{l}\text { Prediction } \\
\text { hardware }\end{array}$ \\
\hline ours & $\left(N+2 l(N)+\left[\log _{3 / 2}\left(\frac{N}{2}\right)\right]-2\right)$ & $24 T_{F A}$ & $37 T_{F A}$ & $51 T_{F A}$ & no & no & simple \\
\hline$[3]$ & $\left(\left[\log _{2} N\right\rceil \times N / 3+4 N / 3\right)$ & $43 T_{F A}$ & $72 T_{F A}$ & $96 T_{F A}$ & yes & no & simple \\
\hline$[4]$ & $5 N / 2$ & $40 T_{F A}$ & $60 T_{F A}$ & $80 T_{F A}$ & yes & no & complex \\
\hline$[5]$ & $\left(N+\left\lceil\left(\log _{3} N\right)-2\right\rceil \times 2\right.$ & $34 T_{F A}$ & $50 T_{F A}$ & $68 T_{F A}$ & no & no & complex \\
\hline$[6]$ & $\left(1.625 N+N / 12+\log _{2} N+1\right)$ & $33 T_{F A}$ & $47 T_{F A}$ & $61 T_{F A}$ & no & $2^{N / 3}$ & simple \\
\hline
\end{tabular}

the input angle. As of area comparison, our proposed ROM-free CSA architecture also requires less hardware complexity compared with the other methods mentioned above.

A 16-bit CORDIC-based sin/cosine generator similar to the architecture in Fig. 1 was synthesized and mapped on the Virtex-300 type FPGA chip. The critical path delay across the entire architecture is less than $75 \mathrm{~ns}$. Compared with the approach in [3], our design achieves more than $25 \%$ improvement in speed performance (due to the parallelization of the sign bit selection), and 30\% saving in hardware cost (due to the elimination of the $\mathrm{Z}$ datapath). Another comparison is made for the ROM-based approach in [6] that calls for 47 configurable logic blocks (CLBs) for the polarity prediction, our proposed sin/cosine generator, with only 28 CLBs for the sign-bit determination, saves more than $40 \%$ hardware cost in the prediction of rotation directions.

\section{CONCLUSION}

We presented a novel recoding method to predict all the directions of CORDIC microrotations and apply it to the gener- ation of sine and cosine functions. The proposed architecture does not need exponentially increased ROM or complicated prediction hardware. The speed is also improved by implementing the microrotation stages using carry-save addition with reduced number of operands.

\section{REFERENCES}

[1] A. Madisetti, Y. Kwentus, and A. N. Willson, Jr., "A 100-MHz, 16-b, direct digital frequency synthesizer with a $100-\mathrm{dBc}$ spurious-free dynamic range," IEEE J. Solid-State Circuits, vol. 34, pp. 1034-1043, Aug. 1999.

[2] J. E. Volder, "The CORDIC trigonometric computing technique," IRE Trans. Electron. Comput., vol. 8, pp. 330-334, Sept. 1959.

[3] S. Wang, V. Piuri, and E. E. Swartzlander, "Hybrid CORDIC algorithms," IEEE Trans. Comput., vol. 46, pp. 1202-1207, Nov. 1997.

[4] D. S. Phatak, "Double step branching CORDIC: A new algorithm for fast sine and cosine generation," IEEE Trans. Comput., vol. 47, pp. 587-602, May 1998.

[5] J. H. Kwak and E. E. Swartzlander, Jr., "A new scheme for prediction of rotation directions in CORDIC processing," in Proc. 42nd Midwest Symp. on Circuits and Systems, 1999, pp. 870-873.

[6] M. Kuhlmann and K. K. Parhi, "P-CORDIC: A precomputation based rotation CORDIC algorithm," EURASIP J. Appl. Signal Process., vol. 2002, pp. 936-943, Sept. 2002. 